\title{
IDENTIFICAÇÃO DE FUNGOS E MICOTOXINAS EM GRÃO DE MILHO
}

Juliana Teodora de Assis Reges ${ }^{1}$, Josiane Walleria Rodrigues ${ }^{2}$, Simone Duarte Ramalho da Silva $^{2}$, Michelle Nogueira de Jesus $^{2}$, Marcelo Henrique de Souza ${ }^{2}$, Iago de Jesus Santos ${ }^{2}$, Samara Moreira do Santos ${ }^{2}$, Sirlene Martins Ribeiro², Thainara Matos Mendes ${ }^{2}$, Alvaro da Silva Franco Junior ${ }^{2}$

\footnotetext{
${ }^{1}$ Doutora do Programa de Pós-Graduação em Agronomia- UNESP, Ilha Solteira (SP) e Docente da Universidade Estadual de Goiás - Campus de Jataí (GO).

${ }^{2}$ Discente do curso de Tecnologia de Alimentos da Universidade Estadual de Goiás - Campus de Jataí (GO).
}

RESUMO: O milho é um produto muito suscetível à contaminação por fungos, muitas vezes facilitada pelas condições de armazenagem dos grãos. O trabalho teve como objetivo identificar, quantificar e avaliar a ocorrência de fungos e a incidência de micotoxinas (aflatoxina e zearalenona) em grãos de milho armazenados e na pós colheita. Foram coletadas quatros amostras, sendo duas em um silo de armazenamento (Silo Bag) de grãos e as outras duas amostras após a colheita do milho em fazendas na cidade de Jataí / GO. De acordo com os resultados obtidos os fungos A. flavus, F. moniliforme, F. roseum, e Penicillium spp foram identificados nas amostras analisadas. Foi constatada a presença de aflatoxinas nos grãos de milho coletado após a colheita, em níveis superiores ao limite oficial $(20 \mu \mathrm{g} / \mathrm{kg})$ permitido pela legislação brasileira.

Palavras-chave: Milho em grãos. Condições de armazenamento. Aflatoxinas. Zearalenona.

\section{IDENTIFICATION OF FUNGI AND MYCOTOXINS IN CORN GRAIN}

\begin{abstract}
Corn is a product very susceptible to fungal contamination often facilitated by the grain storage conditions. The study aimed to identify, quantify and evaluate the occurrence of fungi and the incidence of mycotoxins (aflatoxin and zearalenone) in corn grain stored and post harvest. Four samples were collected, two in a storage silo (Silo Bag) grains and the other two samples after the corn harvest on farms in the city of Jataí / GO. According to the results obtained fungus A. flavus F. moniliforme, F. roseum, Penicillium spp were identified in the samples analyzed. the presence of aflatoxins in corn grains collected after harvest, at levels above the official limit $(20 \mu \mathrm{g} / \mathrm{kg})$ permitted by Brazilian law was found.
\end{abstract}

Key words: Corn grain. Storage conditions. Aflatoxins. Zearalenone.

\section{INTRODUÇÃO}

O setor agrícola tem uma influência na economia brasileira inquestionável. Quando são consideradas todas as transações que ocorrem ao longo das cadeias produtivas do agronegócio, observa-se que em torno de $30 \%$ do que é produzido no Brasil tem origem, de alguma forma, no setor agrícola. Com relação a produção agrícola e a indústria de alimentos, 
com o milho, de acordo com a FAO (2003), apresenta aproximadamente $25 \%$ dos grãos produzidos no mundo tem algum nível de contaminação por fungos produtores de micotoxinas (PEZZINI et al., 2005).

De acordo com Márquez (2008), produtos agrícolas tais como milho, soja aveia, sorgo, arroz, trigo, centeio e outros, são suscetíveis à contaminação por fungos durante as diferentes etapas de produção, como no campo, colheita, durante a secagem, manufatura, transporte e armazenamento. Os fungos pertencentes ao gênero Aspergillus, merece destaque, devido a capacidade de adaptação desse gênero a uma variedade de ambientes e tipos de substratos que pode gerar impactos negativos na economia mundial, pois atuam como agentes degradadores de produtos agrícolas, sendo capazes de produzir micotoxinas, além de algumas espécies, destacarem-se como importantes patógenos em plantas (FRAGA et al., 2008; SILVA, 2012).

Diversos autores tem relatado a ocorrência de micotoxinas nos alimentos que são consumidos no Brasil. Nas últimas décadas, houve um aumento destes fungos em milho que produzem as micotoxinas aflatoxinas e a zearalenoma (ALMEIDA et al., 2002; ORSI et al., 2000; FARIAS et al., 2000). Contudo, o crescimento de fungos e a presença de toxinas não são sinônimos de toxidade, porque nem todos fungos são produtores de micotoxinas. Os gêneros de fungos mais comumente associados e produtores de micotoxinas em grãos, são Aspergillus, Penicillium e Fusarium (IAMANAKA et al., 2010).

Essas micotoxinas podem causar danos à saúde humana, deve-se avaliar a qualidade de grãos que está disponível na região e verificar a adequação de armazenagem e a pós colheita. O presente trabalho teve como objetivo identificar, quantificar e avaliar a ocorrência de fungos e micotoxinas (aflatoxina e zearalenona) em grãos de milho armazenados e na pós colheita, em amostras obtidas de fazendas localizadas em Jataí-GO.

\section{MATERIAL E MÉTODOS}

\section{Material}

Foram coletadas quatros amostras, sendo duas retiradas de um silo de armazenamento de grãos de milho (A, B) e duas amostras logo após a colheita do milho (C, D). Estas amostras foram coletadas em quatro fazendas na cidade de Jataí, GO, em junho de 2015. As amostras do Silo Bag estavam estocadas desde a safra de 2014.

Para as análises das amostras, foram utilizados aproximadamente 1,0 $\mathrm{Kg}$ de grãos que foi acondicionada em frascos plásticos para a quantificação de fungos e micotoxinas (aflatoxinas e zearalenona). Para a identificação e quantificação dos fungos, foram utilizados os grãos de milho inteiros e para as demais determinações os grãos foram moídos, classificados por tamanho de partícula em peneira 20 mesh, submetidas ao quarteamento e analisadas em cinco repetições. $O$ experimento foi desenvolvido no Laboratório da Universidade Estadual Goiás, Campus de Jataí, GO.

Cultura Agronômica, Ilha Solteira, v.25, n.2, p.147-154, 2016 


\section{Detecção e Identificação Fúngica}

Foi utilizado o método adotado por Christensen e Meronuck (1986) para a determinação de fungos presentes nas amostras dos grãos de milho. $\mathrm{O}$ meio de cultura utilizado foi o BDA (ágar (15 g.L $\left.\mathrm{L}^{-1}\right)$, batata dextrose $\left(18,5\right.$ g.L $\left.\mathrm{L}^{-1}\right)$, estreptomicina $\left(0,050\right.$ g.L $\left.\mathrm{L}^{-1}\right)$ e cloranfenicol $\left(0,050\right.$ g. $\left.\left.\mathrm{L}^{-1}\right)\right)$. Foram analisadas 100 grãos por amostragem em quintuplicata, plaqueando-se 20 grãos em cada placa, de modo que fiquem dispostos em 4 fileiras de 5 grãos cada. Os grãos ficaram com o germe ou embrião voltados para cima e na mesma direção, afim de facilitar a avaliação. As placas foram incubadas em estufa a $25^{\circ} \mathrm{C}$, durante 5 dias.

A identificação e quantificação dos fungos foram realizadas com o auxílio de microscópio estereoscópico. A quantificação dos fungos foi submetida à análise de Variância e as médias comparadas por contraste ortogonal entre Silo Bag e pós colheita empregando-se o programa SISVAR (FERREIRA, 2011).

\section{Deteção de Micotoxinas}

A zearalenona foi extraída e quantificada segundo procedimentos descritos por Visconti e Pascale (1998). A quantificação foi realizada em cromatógrafo líquido de alta eficiência com detector de fluorescência (excitação $\lambda=267 \mathrm{~nm}$ e emissão $\lambda=450 \mathrm{~nm}$ ), coluna Agilent Zorbax C18 (5 $\mu \mathrm{m}, 250$ x 4,6 mm), pré-coluna Agilent Zorbax C18 (5 $\mu \mathrm{m}, 25 \mathrm{~mm}$ x 4,6 mm), com fluxo de $1 \mathrm{~mL} /$ minuto. A fase móvel foi composta por água ácida (3\% de ácido acético v/v): metanol (50:50, v/v) e injeção de $50 \mu \mathrm{L}$ de amostra, de acordo com a metodologia de Pezzini et al (2005). A zearalenona foi estimada em uma curva de calibração analítica. A curva da zearalenona foi feita com padrão contendo as concentrações de 1,5; 2,5; 5,0; 10,0 e $15,0 \mu \mathrm{L} / \mathrm{mL}$.

A extração e purificação das aflatoxinas (B1, B2, G1 e G2) foi feita segundo Soares e Rodriguez-Amaya (1989). As aflatoxinas foram identificadas e quantificadas por cromatografia em camada delgada (placas de sílica gel 60 G, Merck), utilizando-se como fase móvel tolueno: acetato de etila: clorofórmio: ácido fórmico (70:50:50:20 v/v/v/v), descrita por Pezzini et al. (2005). As leituras das fluorescências de amostra e padrões foram feitas em densitômetro, a $366 \mathrm{~nm}$ e o cálculo das concentrações foi obtido através das curvas de calibração. A curva das aflatoxinas (AF) foi realizada com os seguintes valores: $A F B 1=1,189$ $\mu \mathrm{g} / \mathrm{mL} ;$ AFB2 $=0,91 \mu \mathrm{g} / \mathrm{mL} ;$ AFG1 $=1,61 \mu \mathrm{g} / \mathrm{mL}$ e AFG2 $=1,39 \mu \mathrm{g} / \mathrm{mL}$. Essas concentrações foram aplicadas nas quantidades de $1,0 \mu \mathrm{L} ; 2,0 \mu \mathrm{L}$ e $5,0 \mu \mathrm{L}$ e as leituras das amostras foram repetidas cinco vezes.

Os limites de detecção de aflatoxinas considerados foram de $1,0 \mu \mathrm{g} / \mathrm{Kg}(85,0 \% \mathrm{de}$ recuperação) e para a zearalenona de $10,0 \mu \mathrm{g} / \mathrm{Kg}$ (80 \% de recuperação) (PEZZINI et al., 2005).

Cultura Agronômica, Ilha Solteira, v.25, n.2, p.147-154, 2016 


\section{RESULTADOS E DISCUSSÃO}

De acordo com os resultados obtidos foi observada a ocorrência de Aspergillus flavus, Fusarium roseum, Fusaruium moniliforme e Penicillium spp (Tabela 1) nas amostras de grãos de milho em silos (A, B) e após a colheita (C, D).

Tabela 1. Crescimento fúngicos (\%) em amostras de milho, em Silos Bag (A e B) e PósColheita (C e D).

\begin{tabular}{lcccc}
\hline Espécie Fúngica & A & B & C & D \\
\hline & & & & \\
Aspergillus flavus $^{*}$ & 30,98 & 65,34 & 25,76 & 46,98 \\
Fusarium roseum $^{*}$ & 13,98 & 11,98 & 39,78 & 35,98 \\
F. moniliforme* $^{*}$ & 11,78 & 14,25 & 28,98 & 47,98 \\
Penicillium spp $^{*}$ & 36,93 & 37,34 & 10,76 & 14,78 \\
\hline
\end{tabular}

O contrate das amostras retiradas do Silo Bag (A; B) diferiram estatisticamente* $(\mathrm{p}<0,01)$ das amostras retiradas após a colheita (B; C).

Na incidência de $A$. flavus apresentou uma variação de 31 a $65 \%$ nas amostras do Silo Bag e de 26 a 47\%, em amostras de milho analisadas após a colheita. As amostras retiradas do Silo Bag diferiram das amostras do campo ( $\mathrm{p}<0,01)$. Atualmente, os grãos ardidos em milho, causado por A. flavus, constituem-se, um dos principais problemas de qualidade dos grãos armazenados, essas perdas qualitativas por grãos ardidos são motivos de desvalorização do produto (FRAGA et al., 2008).

Com relação às percentagens de $F$. roseum houve diferença estatísticas $(\mathrm{p}<0,01)$ entre as amostras do Silo Bag e após a colheita do milho. De acordo com as médias de percentagem em relação a espécie $F$. roseum, pode-se observar uma variação de 11 a 14\% (Silo) e de 35 a 40\% (Pós-Colheita). A espécie fúngica Fusarium spp é considerado um fungo de campo, que infecta os grãos durante o amadurecimento e o dano é causado antes da colheita. Entretanto, não se desenvolvem durante o armazenamento, exceto ocasionalmente em grãos de milho com alto teor de umidade. (PEZZINI et al., 2005).

A incidência de F. moniliforme diferiu do Silo Bag e pós-colheita (11 a $15 \%$ e 28 a $48 \%$ respectivamente). Estes resultados são semelhantes aos obtidos por Marcia e Lazzari (1998), que encontraram maior infestação de $F$. moniliforme em amostras de milho em grãos no campo. A espécie $F$. moniliforme produz micotoxinas cancerígenas, com efeito comprovado, tanto para animais quanto para o homem (COLVIN; HARRISON, 1992; RHEEDER et al., 1992). Dessa maneira, a presença desse fungo em sementes reveste-se de importância especial, não somente devido aos danos econômicos que acarreta, mas também pelo perigo do consumo de grãos infectados e seus derivados (TANAKA, 2001).

De acordo com a média de percentagem da espécie Penicillium spp, já observa-se que houve diferença entre os dois grupos avaliados. O grupo das amostras do Silo Bag (A e B), apresentaram maiores incidência de Penicillium spp em relação às amostras de campo. Diversos fatores podem influenciar o crescimento desse fungo, como, condições temperaturas

Cultura Agronômica, Ilha Solteira, v.25, n.2, p.147-154, 2016 
e umidades, grãos quebrados, presença de insetos, armazenamento inadequado, entre outros (RAMAKRISHNA, 1990).

De acordo com os resultados das análises de aflatoxinas $(\mathrm{B} 1+\mathrm{B} 2+\mathrm{G} 1+\mathrm{G} 2)$ em amostras de grãos de milho, pode-se observar (Tabela 2) que as amostras analisadas após a colheita (C e D) ultrapassou o limite oficial permitido pela Anvisa. Com relação ao limite permitido pelo MS/Anvisa (2011) e pelo MAPA (1996), para alimentos destinados ao consumo humano $(\mathrm{B} 1+\mathrm{B} 2+\mathrm{G} 1+\mathrm{G} 2)$ o limite oficial é de $20 \mu \mathrm{g} / \mathrm{kg}$. Esse limite é comparado aos estabelecidos por outros países e recomendado pela Organização para Alimentação e Agricultura.

Tabela 2. Ocorrência de aflatoxinas B1, B2, G1 e G2 e zearalenona (ZEA) em amostras de grãos de milho retirados do Silo Bag (A e B) e após a colheita (C e D).

\begin{tabular}{|c|c|c|c|c|c|}
\hline \multirow[t]{2}{*}{ Amostras } & \multicolumn{4}{|c|}{ Micotoxinas $(\mu \mathrm{g} / \mathrm{kg})$} & \multirow[b]{2}{*}{ ZEA } \\
\hline & aflatoxina B1 & aflatoxina B2 & aflatoxina $\mathrm{G} 1$ & aflatoxina $\mathrm{G} 2$ & \\
\hline A & 1 & ND & 1 & ND & ND \\
\hline B & 1 & ND & 12 & ND & ND \\
\hline $\mathrm{C}$ & 10 & 12 & 5 & ND & ND \\
\hline $\mathrm{D}$ & 3 & 12 & 10 & ND & ND \\
\hline
\end{tabular}

ND: menor que o limite de detecção.

Limite de detecção: aflatoxina: $1 \mu \mathrm{g} / \mathrm{kg}$; zearalenona: $10 \mu \mathrm{g} / \mathrm{kg}$.

Os níveis de contaminação variaram de 1 a $10 \mu \mathrm{g} / \mathrm{kg}$ de aflatoxina B1, $12 \mu \mathrm{g} / \mathrm{kg}$ de aflatoxina B2, de 1 a $12 \mu \mathrm{g} / \mathrm{kg}$ de aflatoxina G1 e as aflatoxinas G2 e ZEA não foram detectadas. De acordo com Tanaka (2001) e Diniz (2002), o desenvolvimento e a formação das aflatoxinas em alimentos são dependentes de uma série de fatores, principalmente daqueles relacionados à umidade maior que $85 \%$, temperatura entre $8^{\circ} \mathrm{C} \mathrm{e} 42^{\circ} \mathrm{C}$, presença de oxigênio e à constituição do substrato.

Os resultados aqui observados corroboram os de Cortês et al. (2000), quando 28,57\% das amostras de milho retiradas de lavouras do estado de Mato Grosso apresentavam nível médio de aflatoxinas de $12,35 \mu \mathrm{g} / \mathrm{kg}$, o que evidenciou que o milho no ponto de colheita já apresentava a presença de aflatoxinas. Ramos et al. (2008) avaliaram a ocorrência natural das aflatoxinas B1, B2, G1 e G2 em grãos de milho no estado de Goiás (Jataí, Montividiu e Goiânia) e verificaram que os grãos apresentavam aflatoxinas em níveis de 0 a $277,8 \mu \mathrm{g} / \mathrm{kg}$ de B1, de 0 a $14 \mu \mathrm{g} / \mathrm{kg}$ de B2, de 0 a $34,1 \mu \mathrm{g} / \mathrm{kg}$ de $\mathrm{G} 1$, e não foi detectada a aflatoxina $\mathrm{G} 2$. Marques et al. (2009) analisaram a incidência de fungos dos gêneros Aspergillus, Fusarium e Penicillium e as contaminações com micotoxinas em grãos de cinco híbridos comerciais de milho, em função da umidade de colheita, e observaram que a produção de aflatoxinas ocorreu em grãos nas espigas, no campo, devido ás condições ambientais favoráveis ao desenvolvimento do fungo.

Não foi detectada a presença de zearalenona nas amostras de milho analisadas (Tabela 2). Com relação aos valores de aflatoxinas encontrados neste trabalho é preocupação com a qualidade de grãos que são disponibilizadas para a comercialização e para a produção de alimentos, ração de animais, pois essas micotoxinas causam riscos à saúde humana e animal. 


\section{CONCLUSÃO}

A. flavus, $F$. moniliforme, $F$. roseum, e Penicillium spp, foram identificados nos grãos de milho procedentes do Silo Bag e pós-colheita.

A produção de aflatoxinas ocorreram em grãos de milho armazenados após a colheita, em níveis acima do limite oficial permitido pela legislação brasileira.

\section{AGRADECIMENTOS}

A Universidade Estadual Goiás (UEG), pela aprovação do projeto.

\section{REFERÊNCIAS BIBLIOGRÁFICAS}

ALMEIDA, A. P.; FONSECA, H.; FANCELLI, A. L.; DIREITO, G. M.; ORTEGA, E.M.; CORREAA, B. Mycoflora and fumonisin contamination in Brazilian corn from sowing to harvest. Journal Agricultural Food Chemistry, Estados Unidos, v. 50, n. 13, p.3877-3882, 2002.

CHRISTENSEN, C. M.; MERONUCK, R. A. Quality maintenance in stored grains and seeds. Minessota: University of MN Press, 1986. 150 p.

COLVIN, B. M.; HARRISON, L. R. Fumonisin-induced pulmonary edema and hydrothorax in swine. Mycopathologia, Holanda, v. 117, n. 1, p.79-82, 1992.

CORTÊS, N. A.; CASSETARI NETO, D.; CORREA, B. Ocorrência de aflatoxinas em milho produzido pelo sistema tradicional de cultivo, em comunidades de agricultura familiar, no estado de Mato Grosso. Higiene Alimentar, São Paulo, v. 77, n. 14, p.16-25, 2000.

DINIZ, S. P. S. S. Micotoxinas. Campinas: Livraria e Editora Rural, 2002. 181 p.

FARIAS, A. X.; ROBBSS, C. F.; BITTENCOURT, A. M.; ANDERSEN, P. M.; CORRÊA, T. B. S. Contaminação endógena por Aspergillus spp. em milho pós-colheita no Estado do Paraná. Pesquisa Agropecuária Brasileira, Brasília, v. 35, n. 3, p.617-621, 2000.

FAO. Worldwide regulations for micotoxins in food and in feed in 2003. (FAO. Food and Nutrition Paper, 81). Disponível em: http://www.fao.org/docrep/007/y5499e/ y5499e07.htm . Acesso em: 13 Nov. 2015.

FRAGA, M. E.; SANTANA, D. M. N.; GATTI, M. J.; DIREITO, GLORIA. MARIA.; CAVAGLIERI, L. R.; ROSA, CARLOS ALBERTO DA ROCHA. Characterization of Aspergillus species based on fatty acid profiles. Memórias do Instituto Oswaldo Cruz, Rio de Janeiro, v. 103, p.540-544, 2008.

FERREIRA, D. F. Sisvar: a computer statistical analysis system. Ciência e Agrotecnologia, Lavras, v. 35, n. 6, p.1039-1042, 2011.

IAMANAKA, B. T.; TANIWAKI, M. H.; OLIVEIRA, I. S. Micotoxinas em Alimentos. Anais da Academia Pernambucana de Ciência Agronômica, Recife, v. 7, p.138-161, 2010.

Cultura Agronômica, Ilha Solteira, v.25, n.2, p.147-154, 2016 
MÁRCIA, B. A.; LAZZARI, F. A. Monitoramento de fungos em milho em grão, grits e fubá. Ciência Tecnologia Alimentos, Campinas, v. 18, n. 4, p.363-367, 1998.

MÁRQUEZ, R. N. Uso de Adsorbentes de Micotoxinas em La Salud Animal. CenidMicrobiologia, INIFAP México. In SCUSSEL, V. M.; ROCHA, M. W.; LORINI, I. . Atualidades em Micotoxinas e Armazenagem Qualitativa de grãos II. $1^{\circ}$ ed. Florianópolis: ABMAG, 2008. cap. 3, p. 101-105.

MARQUES, O. J.; VIDIGAL-FILHO, P. S.; DALPASQUALE, V. A.; SCAPIM, C. A.; PRICINOTTO, L. F.; MACHINSKI, M. Incidência fúngica e contaminações por micotoxinas em grãos de híbridos comerciais de milho em função da umidade de colheita. Acta Scientiarum Agronomy, Maringá, v. 31, n. 4, p.667- 675, 2009.

MINISTÉRIO DA SAÚDE. Resolução RDC n ${ }^{\circ}$ 7, de 18 de fevereiro de 2011. Dispõe sobre limites máximos tolerados (LMT) para micotoxinas em alimentos. Diário Oficial da União. Brasília, DF, 9 mar. 2011. Seção I, p. 66.

MINISTÉRIO DA AGRICULTURA DO ABASTECIMENTO E DA REFORMA AGRÁRIA. Portaria n. 183, de 21 de março de 1996. Adota Regulamento Técnico do Mercosul sobre limites máximos de aflatoxinas admissíveis no leite, amendoim e milho, aprovado pela Resolução do Grupo Mercado Comum do Sul n. 56/94. Diário Oficial da União. Brasília, DF, 25 mar. 1996, Seção I, p. 4929.

ORSI, R. B.; CORREAA, B.; POZZI, C. R.; SCHAMMASS, E. A.; NOGUEIRA, J. R.; DIAS, S. M. C.; MALLOZZI, M. A. B. Mycoflora and occurrence of fumonisis in freshly harvested and stored hybrid mayze. Journal of Stored Products Research, Elmsford, v. 36, n. 1, p.75$87,2000$.

PEZZINI, V.; VALDUGA, E.; CANSIAN, R. L. Incidência de fungos e micotoxinas em grãos de milho armazenados sob diferentes condições. Revista Instituto Adolfo Lutz, São Paulo, v. 64, n. 1, p.91-96, 2005.

RAMAKRISHNA, Y.; RAMESH, V. B.; VASANTHI S. Natural occurrence of mycotoxins in staple foods in Índia. Jornal of Agricultural and Food Chemistry, Estados Unidos, v. 38, n. 9, p. 1857-1859, 1990.

RAMOS, C. R. B. A.; BRASIL, E. M.; GERALDINE, R. M. Contaminação por aflatoxinas em híbridos de milho cultivados em três regiões do estado de Goiás. Pesquisa Agropecuária Tropical, Goiânia, v. 38, n. 2, p.95-102, 2008.

RHEEDER, J. P.; MARASAS, W. F. O.; THIEL, P. G.; SYDENHAM, E. E.; SHEPHARD, G. S.; VAN SCHALKWYK, D. J. Fusarium moniliforme and fumonisins in corn in relation to human esophageal cancer in Transkei. Phytopathology, St. Paul, v. 82, n. 3, p.353-357, 1992.

SCUSSEL, V. M.; ROCHA, M. W.; LORINI, I. Atualidades em Micotoxinas e Armazenagem Qualitativa de grãos II. $1^{\text {o }}$ ed. Florianópolis: ABMAG, 2008. cap. 3, p. 101 105.

Cultura Agronômica, Ilha Solteira, v.25, n.2, p.147-154, 2016 
SILVA, J. L. Identificação Morfológica e Molecular de Aspergillus flavus e Aspergillus parasiticus isolados de alimentos destinados a caprinos em lactação. 2012. 85 p. Dissertação (Mestrado em Sanidade, Segurança Alimentar e Ambiental no Agronegócio) Instituto Biológico - Agência Paulista de Tecnologia dos Agronegócios, São Paulo, 2012.

SOARES, L. M.; RODRIGUEZ-AMAYA, D. B. Survey of aflatoxins, achratoxin A, zearalenone and sterigmatocystin in brasilian foods by using multitoxin layer chromatographic methods. Journal Association of Official Analytical Chemists, Arlington, v. 72, n. 1, p.22-26, 1989.

TANAKA, M. A. S. Sobrevivência de Fusarium moniliforme em sementes de milho mantidas em duas condições de armazenamento. Fitopatologia Brasileira, Brasília, v. 26, n. 1, p.6064, 2001.

VISCONTI, A.; PASCALE, M. Determination of zearalenone in corn by means of immnoaffinity clean-up and high-performance liquid chromatography with fluorescence detection. Jornal of Chromatography A, Estados Unidos, v. 815, n. 1, p.133-40, 1998. 